\title{
Comparison of volatile flavor compounds of Artemisia annua L. extracted by simultaneous steam distillation extraction and solid-phase micro extraction
}

\author{
Young Shin Hong, Ji Hyeon Son, Ji Young Jeong, Ok Yeon Song, Jin Ha Lee, \\ Kyoung Su Kim* \\ Department of Food and Nutrition, Chosun University, Gwangju 61452, Korea
}

\section{SDE 및 SPME법을 이용한 개똥숙(Artemisia annua L.)의 휘발성 향기성분의 비교}

\author{
홍영신 · 손지현 · 정지영 · 송옥연 · 이진하·김경수* \\ 조선대학교 식품영양학
}

\begin{abstract}
This study was conducted to compare the volatile flavor compounds of Artemisia annua L. after extraction by simultaneous steam distillation extraction (SDE) and solid-phase micro extraction (SPME) followed by gas chromatography-mass spectrometry (GC-MS) analysis. Via SDE and SPME processes, $79(1,254.00 \mathrm{mg} / \mathrm{kg}) \mathrm{and}$ $39(488.74 \mathrm{mg} / \mathrm{kg})$ compounds were identified respectively. The compounds extracted by SDE included 27 alcohols, 13 aldehydes, 22 hydrocarbons, 3 esters, 12 ketones, 1 oxide and $1 \mathrm{~N}$-containing compound, on the other hand, using the SPME method, 7 alcohols, 5 aldehydes, 1 ester, 18 hydrocarbons, 7 ketones, and 1 oxide were extracted. The major volatile flavor compounds of Artemisia annua $\mathbf{L}$. isolated by the two methods were caryophyllene oxide, $\beta$-caryophyllene, camphor, $\beta$-selinene, $\gamma$-muurolene, 1,8-cineol, (E)-pinocarveol and pinocarvone. The sesquiterpene named caryophyllene oxide was the most abundant volatile flavor compound with relative contents of $234.16 \mathrm{mg} / \mathrm{kg}$ and $195.44 \mathrm{mg} / \mathrm{kg}$ obtained by the SDE and SPME methods, respectively. Among the identified volatiles, sabinene, $\beta$-pinene, a-terpinene, $\mathrm{\gamma}$-terpinene, yomogi alcohol, myrtenol, (Z)-nerolidol, p-cymen-8-ol and eugenol were detected by the SDE method only while (E)-anethole and a-cubebene were detected by the SPME method only. This study confirmed that the composition and contents of the volatile flavor compounds vary between different extraction methods. More volatile flavor compounds were identified using the SDE method than the SPME method.
\end{abstract}

Key words : Artemisia annua L., volatile flavor compounds, SDE, SPME, GC-MS

\section{서 론}

국화과(Compositae)에 속하는 쑥(Artemisia species)은 적 응력이 강하고 번식능력이 뛰어나며 세계적으로 약 2,000 여종의 다양한 품종이 널리 분포되어 있다 $(1,2)$. 쑥의 잎은

*Corresponding author. E-mail : kskim@chosun.ac.kr Phone : 82-62-230-7724, Fax : 82-62-224-8880

Received 1 June 2018; Revised 15 June 2018; Accepted 20 June 2018.

Copyright (c) The Korean Society of Food Preservation. All rights reserved.
어긋나게 돋아나고 작은 톱니가 있으며 뒷면에는 회백색의 작은 털이 빽빽한 형태를 지니고 있다. 쑥의 정유 성분은 다양한 휘발성 향기성분을 포함하고 있으며, 항균, 살충 및 항종양 등의 생리 활성 기능을 가지는 다량의 폴리페놀 화합물이 함유된 것으로 알려져 있다(3).

개똥쑥(Artemisia annua L.)은 일년생 초본으로 한방에서 황화호(黃花高) 또는 청호(菁高)라고 불리며 우리나라를 비 롯한 세계 각국에 분포되어 있다. 주로 강가나 길가에서 $1 \mathrm{~m}$ 정도 자라나며 초여름부터 녹황색 꽃을 피우는 것이 특징이다. 또한 개똥쑥은 개사철쑥의 전초로서 꽃이 피기 전 그늘에 말려 사용하는데, 강하고 특이한 향으로 인해 
다른 쑥속과 확연히 구별된다(4).

개똥쑥은 중의약에서 오랜 역사 동안 열병, 해열제 및 피부병 치료 등에 사용되었다. 개똥쑥의 줄기 추출물은 단 당류의 생성을 억제하여 급격한 혈당 상승을 낮추고, 수용 성추출액이나 휘발성물질은 곰팡이의 생장을 억제하여 항 균효과를 가진다 $(5,6)$. 개똥쑥의 성분 중 하나인 artemisinin 은 말라리아 치료에 사용되며 병원성을 갖는 원충에 강력한 살생력을 지니고, 포유동물과 초식곤충에게도 기피 작용을 한다(7). 또한 유방암세포를 효과적으로 사멸시키고 암세 포의 증식을 억제하여 높은 항암효과를 보인다는 연구결과 가 보고되고 있다(8).

쑥속의 향기성분 연구로 Choi 등(9)은 eucalyptol, $\beta$ -thujone, borneol, caryophyllene, camphor 등을 확인하였으 며 Jang 등(10)은 $\beta$-myrcene, camphor, endo-borneol, a -thujone, 1,8-cineole 및 (Z)-limonene oxide 등을 확인하였 다. 기존 연구 중 개똥쑥의 약리작용과 효능에 대한 연구결 과들과, 쑥속의 향기성분 연구들은 많으나 추출방법에 따 른 개똥쑥의 휘발성 향기 성분 비교연구는 미흡한 실정이 다.

휘발성 향기 성분의 추출방법은 연속수증기증류 (simultaneous steam distillation extraction, $\mathrm{SDE}$ )법과 고체상 미량분석(solid phase micro extraction, SPME)법이 있다. 연 속수증기증류(SDE)법은 다양한 휘발성 유기화합물을 포 집할 수 있는 장점이 있지만, 고온에서 오랜 시간 추출로 인하여 주요 성분이 열에 의해서 변성될 수 있는 단점이 있어 열에 안정된 시료에 주로 이용한다(11). SPME법은 열에 의한 변성이 적으며 적은 양의 시료를 간단히 전처리 할 수 있고, 향기성분 흡착이 가능한 fiber를 사용하여 간단 하고 신속한 분석이 가능하다 $(12,13)$. 또한 시료의 특성에 따라 fiber의 종류, 추출시간 및 추출온도를 변화시켜 선택 적으로 추출할 수 있기 때문에 다양한 분석법 개발에 이용 할 수 있는 장점이 있다(11). 따라서 본 연구는 일반적으로 주로 사용되고 있는 $\mathrm{SDE}$ 및 $\mathrm{SPME}$ 법을 이용하여 개똥쑥의 휘발성 향기성분을 추출한 후 분석하여 추출방법의 효율성 및 휘발성 향기성분을 비교하고자 수행하였다.

\section{재료 및 방법}

\section{재 료}

개똥쑥(Artemisia annua L.)은 2018년 2월 광주광역시 동 구 계림동에 위치한 재래시장에서 자연 건조된 시료 $2 \mathrm{~kg}$ 을 구입하였다. 시료는 blender(MR 350CA, Braun, Spain)로 균질화시켜 $-70^{\circ} \mathrm{C}$ 에 저장하면서 시료로 사용하였다.

시 약

연구에서 사용된 시약인 n-pentane 및 diethyl ether(Fisher
Scientific, Waltham, MA, USA)는 HPLC grade를 구입해 wire spiral packed double distilling apparatus(Nomschliff Geratebau, Wertheim Gemany)으로 재증류한 후 향기성분 추출에 사용하였다. 물은 순수재증류장치(Millipore Corporation, Bedford, MA, USA)에서 얻은 이온교환수(ultra pure water)를 사용하였으며, 무수황산나트륨(DC Chemical Co., Ltd., Seoul, Korea)은 $650^{\circ} \mathrm{C}$ 의 조건에서 회화시킨 후 desiccator에서 방냉하여 유기용매 탈수에 사용하였다.

\section{$\mathrm{SDE}$ 을 이용한 휘발성 향기성분 추출}

균질화 시킨 개똥쑥 시료 $30 \mathrm{~g}$ 을 Milli $\mathrm{Q}$ water $1 \mathrm{~L}$ 와 혼합하고 $5 \mathrm{~N} \mathrm{NaOH}$ 와 $5 \mathrm{~N} \mathrm{HCl}$ 을 이용하여 $\mathrm{pH}$ meter(PSM11R-090, Thermo, Waltham, MA, USA)로 pH 7.0 이 되도록 조정한 후 휘발성 향기성분 추출용 시료로 사용 하였다. 휘발성 향기성분 추출은 Schultz 등(14)에 의해 개 량된 연속수증기증류추출장치(simultaneous steam distillation and extraction apparatus, SDE, Normschliff Weräatebau, Germany)를 이용하였고 정량분석을 위한 내부표준물질로 $100 \mathrm{ppm}$ n-butylbenzene $5 \mathrm{~mL}$ 를 추출 시료에 첨가하였다. 추출용매는 정제시킨 n-pentane:diethyl ether(1:1, v/v)를 100 $\mathrm{mL}$ 로 제조한 후 상압하에서 3시간 동안 추출하였다. 수득 된 추출물에 무수황산나트륨을 첨가하여 수분을 제거해주 었으며, 유기용매 분획분은 vigreux column $(250 \mathrm{~mL}$, Normschliff geratebau, Wertheim, Germany)을 사용하여 농 축시킨 후, $\mathrm{N}_{2}$ 을 이용해 $0.5 \mathrm{~mL}$ 까지 재 농축시켜 GC-MS로 분석하였다(15).

\section{SPME를 이용한 휘발성 향기성분 추출}

균질화된 개똥쑥을 약 $2 \mathrm{~g}$ 칭량하여 headspace glass vial 에 넣은 후 내부표준물질로 지정한 $150 \mathrm{ppm}$ n-butylbenzene 을 $50 \mu \mathrm{L}$ 주입하였다. 시료가 담긴 headspace용 vial을 $75^{\circ} \mathrm{C}$ 로 30 분간 평형상태에 도달시킨 후 5분간 SPME fiber $(\mathrm{DVB} / \mathrm{CAR} / \mathrm{PDMS})$ 를 노출시켜 휘발성 향기성분을 흡착하 여 추출하였고, $250^{\circ} \mathrm{C} \mathrm{GC-MS} \mathrm{injector에서} 5$ 분간 탈착시켜 휘발성 향기성분을 분석하였다.

\section{휘발성 향기성분 분석}

농축시킨 추출액은 GC-MS(gas chromatography-mass spectrometry)를 이용하여 분석하였다. GC-MS는 GCMSQP2010Plus(Shimadzu, Kyoto, Japan) 기기를 사용하였다. Column은 DB-5(60 $\mathrm{mm} \times 0.25 \mathrm{~mm}$ i.d., $0.25 \mu \mathrm{m}$ film thickness, J\&W, CA, USA)를 이용하였다. Temperature program은 $40^{\circ} \mathrm{C}$ 에서 5 분간 머무른 뒤, $2^{\circ} \mathrm{C} / \mathrm{min}$ 의 속도로 $280^{\circ} \mathrm{C}$ 까지 승온시킨 후 10 분간 유지하였다. Injector 온도는 $250^{\circ} \mathrm{C}$ 로 설정하였다. Carrier gas는 $\mathrm{He}$ 을 사용하였고 유속 은 $1.0 \mathrm{~mL} / \mathrm{min}$ 으로 하였으며, injection volume은 $1 \mu \mathrm{L}$ 를 split ratio 1:20로 주입하였다(Table 1). n-Alkane 표준물질인 
$\mathrm{C}_{8}$ 부터 $\mathrm{C}_{20}$ 까지를 이용해 retention index를 구하여 휘발성 향기성분 화합물을 동정하였다(15).

Table 1. GC-MS conditions for identification of volatile flavor compounds

\begin{tabular}{cc}
\hline GC-MS & GCMS-QP2010Plus (Shimadzu, Kyoto, Japan) \\
\hline Column & DB-5(60 mm $\times 0.25 \mathrm{~mm}$ i.d., $0.25 \mu \mathrm{m}$ film thickness $)$ \\
Carrier gas & $\mathrm{He}$ (Flow rate: $1.0 \mathrm{~mL} / \mathrm{min})$ \\
Oven temp program & $40^{\circ} \mathrm{C}(5 \mathrm{~min})-2^{\circ} \mathrm{C} / \mathrm{min}-280^{\circ} \mathrm{C}(10 \mathrm{~min})$ \\
Injector temp & $250^{\circ} \mathrm{C}$ \\
Dectector temp & $300^{\circ} \mathrm{C}$ \\
Ionization voltage & $70 \mathrm{eV}$ \\
Injector volume & $1 \mu \mathrm{L}$ \\
Split ratio & $1: 20$ \\
\hline
\end{tabular}

\section{$\mathrm{GC}-\mathrm{MS}$ 에 의한 휘발성향기 성분 동정}

GC-MS(GC-2010, Shimadzu, Kyoto, Japan)를 이용해 ionization voltage를 $70 \mathrm{eV}$ 로 설정해주었다. Total ionization chromatogram(TIC)에 의한 peak의 성분분석은 mass spectrum library(FFNSC2, NIST14, WILEY7)와 문헌상의 retention index 및 $\mathrm{GC} / \mathrm{MS}$ 분석에 의한 retention index와 일치, 표준물질의 분석 data를 비교하여 확인하였다(15). 동정된 휘발성 향기성분의 함량은 내부표준물질로 첨가된 n-butyl benzene과 확인된 휘발성 향기성분의 peak area를 비교하여 정량하였다.

\section{결과 및 고찰}

개똥쑥의 휘발성 향기성분을 SDE 및 SPME 방법을 이용 하여 추출한 후 $\mathrm{GC} / \mathrm{MS}$ 로 분석하였으며, 동정된 화합물의 chromatogram은 Fig. 1 과 같다. 동정된 휘발성 화합물의 함량과 상대적 농도를 Table 2에 나타내었다.

\section{SDE 방법을 이용한 개똥쑥의 휘발성 향기성분}

$\mathrm{SDE}$ 방법으로 추출한 후 확인된 개똥쑥의 휘발성 향기 성분은 총 79종이며, 함량은 $1,254.00 \mathrm{mg} / \mathrm{kg}$ 으로 확인되었 다. 개똥쑥에서 확인된 휘발성 향기성분은 hydrocarbon류 가 22종(27.85\%)으로 가장 높은 peak area를 보였으며, 그 다음으로는 alcohol류 27종(26.81\%), oxide류 1종(18.67\%), ketone류 12종(18.62\%), ester류 3종(2.09\%), aldehyde류 13 종 $(2.06 \%)$ 및 N-containing compound 1 종 $(0.62 \%)$ 이 확인되 었다(Table 3). 개똥쑥의 주요 휘발성 향기성분은 caryophyllene oxide(18.67\%)로 함량은 $234.16 \mathrm{mg} / \mathrm{kg}$ 으로 가장 높게 확인 되었으며, 다음으로 camphor(10.58\%), $\beta$-caryophyllene(7.74\%), (E)-pinocarveol(5.23\%), $\beta$-selinene(4.16\%), borneol(4.11\%), $\gamma$-muurolene(3.76\%), pinocarvone(3.28\%) 및 1,8-cineole
(2.54\%) 등 순으로 확인되었다. Caryophyllene oxide는 sesquiterpene으로 sweet, fruity, sawdust 및 herbal 특성을 가지며, 식품과 화장품 및 약품의 보존제로 이용되고 있으 며 항균효과도 가지고 있다고 보고된 바 있다(16,17). 또한 Sencsh 등(17)은 칼슘 및 칼륨 대사통로에 긍정적인 영향을 준다고 발표하였다. Camphor는 쑥의 주요 향기성분으로 dry, green 및 leafy한 특성으로 민트향과 비슷한 청량감을 가지며, 1,8-cineol과 함께 유전자의 돌연변이 억제 작용을 나타낸다(18). 1,8-Cineol은 유칼립투스의 에센셜 오일의 주 요 성분으로 약 $90 \%$ 정도 함유되어 있으며, monoterpene alcohol로 eucalyptol로 불리고 있다(19). Caryophyllene은 주 로 정향, 레몬, 자몽 및 라벤더 등에 다량 함유된 성분으로 musty, green, spicy, woody, fruity 및 sweet한 향취를 가지며, 향을 오랫동안 지속시켜줄 수 있어 검은 후추 및 껌의 제조 등 향료 산업에 널리 이용되고 있다(20,21).

Fabien 등(22)은 개똥쑥의 에센셜 오일의 성분으로 1,8-cineole, p-cymene, linalool, camphor, thujone 등을 보고 하였으나, 본 연구에서는 linalool 및 thujone은 검출되지 않았다.

Herman 등(23)의 연구에서 쑥의 주요 향기성분으로 artemisia ketone(63.9\%), artemisia alcohol(7.5\%), myrcene (5.1\%), a-guaiene(4.7\%) 및 camphor(3.3\%) 등이 보고되었 으나, 본 실험에서는 artemisia ketone, myrcene 및 a-guaiene 은 확인되지 않았으며, camphor 및 artemisia alcohol이 각각 $10.58 \%$ 및 $0.30 \%$ 로 동정되었다. Khangholi 등(24)은 개똥쑥 의 에센셜 오일의 주성분이 건조 온도와 추출 조건에 의해 차이가 있다고 보고하였는데, 상온 및 $45^{\circ} \mathrm{C}$ 에서 건조 시 attemisia ketone 및 1,8-cineol이 주성분으로 확인되었으며, $55^{\circ} \mathrm{C}$ 에서 attemisia ketone, 1,8 -cineol 및 camphor, $65^{\circ} \mathrm{C}$ 에서 $\beta$-caryophyllene 및 germacrene $\mathrm{D}$ 가 주요 성분임을 확인하 였다. 위의 연구결과와 같이 본 연구에서도 $\beta$-caryophyllene 과 germacrene $\mathrm{D}$ 의 area\%가 각각 $7.74 \%$ 및 $2.13 \%$ 로 동정되 었는데, 이는 자연건조된 개똥쑥을 다시 $60^{\circ} \mathrm{C}$ 로 재건조하 여 시료로 사용하였기 때문이라고 판단된다. Artemisia ketone은 주로 중국, 세르비아, 보스니아, 이란, 미국 및 프랑 스 지역에서 자생하는 개똥쑥의 에센셜 오일에서 동정된 바 있으며(25), 국내에서는 전북 완주군 대둔산에서 수집된 국화과 쑥속에 속하는 더위지기 정유 성분으로 확인된 바 있다(26). 또 다른 연구에서 Hwang 등(27)은 한국에서 자생 하는 개똥쑥의 주요 에센셜 오일 성분으로 camphor, 1,8-cineol, sabinene, a-pinene, $\beta$-farnesene, caryophyllene, germacrene $\mathrm{D}$ 및 $\beta$-selinene 등이 동정된 바 있는데, 본 연구 에서도 이 화합물들이 모두 확인되었다.

\section{SPME방법을 이용한 개똥쑥의 휘발성 향기성분}

$\mathrm{SPME}$ 방법으로 휘발성 향기성분을 추출한 후 GC-MS로 동정된 개똥쑥의 휘발성 향기성분은 총 39 종으로 함량은 
Table 2. Volatile flavor compounds identified in Artemisia annua L. by simultaneous steam distillation extraction (SDE) and solid phase microextraction (SPME)

\begin{tabular}{|c|c|c|c|c|c|c|c|c|}
\hline \multirow{2}{*}{ No } & \multirow{2}{*}{ R. I } & \multirow{2}{*}{ Name } & \multirow{2}{*}{$\mathrm{MF}^{2)}$} & \multirow{2}{*}{ M.W ( $^{3}$} & \multicolumn{2}{|c|}{ SDE } & \multicolumn{2}{|c|}{ SPME } \\
\hline & & & & & $(\mathrm{mg} / \mathrm{kg})$ & (Area \%) & $(\mathrm{mg} / \mathrm{kg})$ & (Area \%) \\
\hline 1 & 706 & 3-Methylbutanal & $\mathrm{C}_{5} \mathrm{H}_{10} \mathrm{O}$ & 87 & 0.68 & 0.05 & - & - \\
\hline 2 & 719 & 1-Pentene-3-ol & $\mathrm{C}_{5} \mathrm{H}_{10} \mathrm{O}$ & 86 & 0.59 & 0.05 & - & - \\
\hline 3 & 727 & Pentanal & $\mathrm{C}_{5} \mathrm{H}_{10} \mathrm{O}$ & 86 & 0.62 & 0.05 & - & - \\
\hline 4 & 729 & 2-Ethylfuran & $\mathrm{C}_{6} \mathrm{H}_{8} \mathrm{O}$ & 96 & 1.07 & 0.09 & - & - \\
\hline 5 & 751 & 3-Methyl-1-butanol & $\mathrm{C}_{5} \mathrm{H}_{12} \mathrm{O}$ & 88 & 0.42 & 0.03 & - & - \\
\hline 6 & 754 & 2-Methyl-1-butanol & $\mathrm{C}_{5} \mathrm{H}_{12} \mathrm{O}$ & 88 & 0.40 & 0.03 & - & - \\
\hline 7 & 767 & (E)-2-Pentenal & $\mathrm{C}_{5} \mathrm{H}_{8} \mathrm{O}$ & 84 & 0.25 & 0.02 & - & - \\
\hline 8 & 775 & 1-Pentanol & $\mathrm{C}_{5} \mathrm{H}_{12} \mathrm{O}$ & 88 & 1.00 & 0.08 & - & - \\
\hline 9 & 779 & 2-Penten-1-ol & $\mathrm{C}_{5} \mathrm{H}_{10} \mathrm{O}$ & 86 & 0.35 & 0.03 & - & - \\
\hline 10 & 790 & 3-Hexanone & $\mathrm{C}_{6} \mathrm{H}_{12} \mathrm{O}$ & 100 & 0.21 & 0.02 & - & - \\
\hline 11 & 794 & 2-Hexanone & $\mathrm{C}_{6} \mathrm{H}_{12} \mathrm{O}$ & 100 & 0.71 & 0.06 & - & - \\
\hline 12 & 798 & 3-Hexanol & $\mathrm{C}_{6} \mathrm{H}_{14} \mathrm{O}$ & 102 & 0.41 & 0.03 & - & - \\
\hline 13 & 802 & Hexanal & $\mathrm{C}_{6} \mathrm{H}_{12} \mathrm{O}$ & 100 & 6.52 & 0.52 & 1.40 & 0.29 \\
\hline 14 & 836 & Furfural & $\mathrm{C}_{5} \mathrm{H}_{4} \mathrm{O}_{2}$ & 96 & 0.74 & 0.06 & - & - \\
\hline 15 & 855 & (E)-2-Hexenal & $\mathrm{C}_{6} \mathrm{H}_{10} \mathrm{O}$ & 98 & 3.64 & 0.29 & - & - \\
\hline 16 & 857 & (Z)-3-Hexen-1-ol & $\mathrm{C}_{6} \mathrm{H}_{12} \mathrm{O}$ & 100 & 5.57 & 0.44 & - & - \\
\hline 17 & 871 & 1-Hexanol & $\mathrm{C}_{6} \mathrm{H}_{14} \mathrm{O}$ & 102 & 1.23 & 0.10 & - & - \\
\hline 18 & 880 & Heptanal & $\mathrm{C}_{7} \mathrm{H}_{14} \mathrm{O}$ & 114 & 0.46 & 0.04 & - & - \\
\hline 19 & 894 & 2-Heptanone & $\mathrm{C}_{7} \mathrm{H}_{14} \mathrm{O}$ & 114 & 0.74 & 0.06 & - & - \\
\hline 20 & 912 & 2,4-Hexadienal & $\mathrm{C}_{6} \mathrm{H}_{8} \mathrm{O}$ & 96 & 0.26 & 0.02 & - & - \\
\hline 21 & 928 & a-Thujene & $\mathrm{C}_{10} \mathrm{H}_{16}$ & 136 & 1.17 & 0.09 & 0.45 & 0.09 \\
\hline 22 & 938 & a-Pinene & $\mathrm{C}_{10} \mathrm{H}_{16}$ & 136 & 9.44 & 0.75 & 3.06 & 0.63 \\
\hline 23 & 950 & Camphene & $\mathrm{C}_{10} \mathrm{H}_{16}$ & 136 & 6.55 & 0.52 & 2.82 & 0.58 \\
\hline 24 & 956 & 2,4-Thujadiene & $\mathrm{C}_{10} \mathrm{H}_{14}$ & 134 & 1.16 & 0.09 & 0.40 & 0.08 \\
\hline 25 & 960 & (E)-2-Heptenal & $\mathrm{C}_{7} \mathrm{H}_{12} \mathrm{O}$ & 112 & 1.01 & 0.08 & - & - \\
\hline 26 & 965 & Benzaldehyde & $\mathrm{C}_{7} \mathrm{H}_{6} \mathrm{O}$ & 106 & 5.79 & 0.46 & 1.73 & 0.35 \\
\hline 27 & 975 & Sabinene & $\mathrm{C}_{10} \mathrm{H}_{16}$ & 136 & 1.12 & 0.09 & - & - \\
\hline 28 & 979 & $\beta$-Pinene & $\mathrm{C}_{10} \mathrm{H}_{16}$ & 136 & 1.41 & 0.11 & - & - \\
\hline 29 & 1001 & Yomogi alcohol & $\mathrm{C}_{10} \mathrm{H}_{18}$ & 154 & 8.04 & 0.64 & - & - \\
\hline 30 & 1015 & (E,E)-2,4-Heptadienal & $\mathrm{C}_{7} \mathrm{H}_{10} \mathrm{O}$ & 110 & 1.05 & 0.08 & 0.94 & 0.19 \\
\hline 31 & 1019 & a-Terpinene & $\mathrm{C}_{10} \mathrm{H}_{16}$ & 136 & 3.92 & 0.31 & - & - \\
\hline 32 & 1027 & p-Cymene & $\mathrm{C}_{10} \mathrm{H}_{14}$ & 134 & 4.66 & 0.37 & 1.55 & 0.32 \\
\hline 33 & 1031 & Limonene & $\mathrm{C}_{10} \mathrm{H}_{16}$ & 136 & 2.22 & 0.18 & 0.75 & 0.15 \\
\hline 34 & 1035 & 1,8-Cineole & $\mathrm{C}_{10} \mathrm{H}_{18} \mathrm{O}$ & 154 & 31.91 & 2.54 & 14.20 & 2.91 \\
\hline 35 & 1040 & Benzyl alcohol & $\mathrm{C}_{7} \mathrm{H}_{8} \mathrm{O}$ & 108 & 2.12 & 0.17 & - & - \\
\hline I. $S^{4)}$ & 1059 & Buthyl benzene & $\mathrm{C}_{10} \mathrm{H}_{14}$ & 134 & - & - & - & - \\
\hline 36 & 1062 & z-Terpinene & $\mathrm{C}_{10} \mathrm{H}_{16}$ & 136 & 8.08 & 0.64 & - & - \\
\hline 37 & 1071 & (Z)-Sabinene hydrate & $\mathrm{C}_{10} \mathrm{H}_{18} \mathrm{O}$ & 154 & 9.23 & 0.74 & - & - \\
\hline 38 & 1074 & 1-Octanol & $\mathrm{C}_{8} \mathrm{H}_{18} \mathrm{O}$ & 130 & 0.83 & 0.07 & - & - \\
\hline 39 & 1087 & Artemisia alcohol & $\mathrm{C}_{10} \mathrm{H}_{18} \mathrm{O}$ & 154 & 3.73 & 0.30 & - & - \\
\hline 40 & 1092 & a-Terpinolene & $\mathrm{C}_{10} \mathrm{H}_{16}$ & 154 & 4.50 & 0.36 & - & - \\
\hline
\end{tabular}


Table 2. Continued

\begin{tabular}{|c|c|c|c|c|c|c|c|c|}
\hline \multirow{2}{*}{ No } & \multirow{2}{*}{ R.I ${ }^{1)}$} & \multirow{2}{*}{ Name } & \multirow{2}{*}{ M.F ${ }^{2)}$} & \multirow{2}{*}{ M.W W $^{3}$} & \multicolumn{2}{|c|}{ SDE } & \multicolumn{2}{|c|}{ SPME } \\
\hline & & & & & $(\mathrm{mg} / \mathrm{kg})$ & $($ Area \%) & $(\mathrm{mg} / \mathrm{kg})$ & $($ Area \%) \\
\hline 41 & 1103 & (E)-Sabinene hydrate & $\mathrm{C}_{10} \mathrm{H}_{18} \mathrm{O}$ & 154 & 14.07 & 1.12 & 0.74 & 0.15 \\
\hline 42 & 1107 & Nonanal & $\mathrm{C}_{9} \mathrm{H}_{18} \mathrm{O}$ & 142 & 2.23 & 0.18 & 0.54 & 0.11 \\
\hline 43 & 1111 & 6-Methyl-3,5-hdptadien-2-one & $\mathrm{C}_{8} \mathrm{H}_{12} \mathrm{O}$ & 124 & 1.49 & 0.12 & - & - \\
\hline 44 & 1120 & Phenethyl alcohol & $\mathrm{C}_{8} \mathrm{H}_{10} \mathrm{O}$ & 122 & 9.25 & 0.74 & - & - \\
\hline 45 & 1132 & a-Campholenal & $\mathrm{C}_{10} \mathrm{H}_{16} \mathrm{O}$ & 152 & 2.65 & 0.21 & - & - \\
\hline 46 & 1147 & (E)-Pinocarveol & $\mathrm{C}_{10} \mathrm{H}_{16} \mathrm{O}$ & 152 & 65.55 & 5.23 & 7.53 & 1.54 \\
\hline 47 & 1153 & Camphor & $\mathrm{C}_{10} \mathrm{H}_{16} \mathrm{O}$ & 152 & 132.61 & 10.58 & 68.93 & 14.10 \\
\hline 48 & 1156 & Camphene hydrate & $\mathrm{C}_{10} \mathrm{H}_{18} \mathrm{O}$ & 154 & 2.91 & 0.23 & - & - \\
\hline 49 & 1169 & (Z)-Chrysanthenol & $\mathrm{C}_{10} \mathrm{H}_{16} \mathrm{O}$ & 152 & 4.66 & 0.37 & - & - \\
\hline 50 & 1170 & Pinocarvone & $\mathrm{C}_{10} \mathrm{H}_{14} \mathrm{O}$ & 150 & 41.10 & 3.28 & 7.27 & 1.49 \\
\hline 51 & 1173 & Borneol & $\mathrm{C}_{10} \mathrm{H}_{18} \mathrm{O}$ & 154 & 51.57 & 4.11 & 6.46 & 1.32 \\
\hline 52 & 1179 & Menthol & $\mathrm{C}_{10} \mathrm{H}_{20} \mathrm{O}$ & 156 & 10.91 & 0.87 & 1.33 & 0.27 \\
\hline 53 & 1185 & 4-Terpineol & $\mathrm{C}_{10} \mathrm{H}_{18} \mathrm{O}$ & 154 & 29.40 & 2.34 & 2.33 & 0.48 \\
\hline 54 & 1192 & p-Cymen-8-ol & $\mathrm{C}_{10} \mathrm{H}_{14} \mathrm{O}$ & 150 & 13.94 & 1.11 & - & - \\
\hline 55 & 1197 & a-Terpineol & $\mathrm{C}_{10} \mathrm{H}_{18} \mathrm{O}$ & 154 & 6.70 & 0.53 & - & - \\
\hline 56 & 1206 & Myrtenol & $\mathrm{C}_{10} \mathrm{H}_{16} \mathrm{O}$ & 152 & 25.27 & 2.02 & - & - \\
\hline 57 & 1219 & Vervenone & $\mathrm{C}_{10} \mathrm{H}_{14} \mathrm{O}$ & 150 & 13.18 & 1.05 & 1.57 & 0.32 \\
\hline 58 & 1226 & (E)-Carveol & $\mathrm{C}_{10} \mathrm{H}_{16} \mathrm{O}$ & 152 & 15.01 & 1.20 & 0.88 & 0.18 \\
\hline 59 & 1238 & (Z)-Carveol & $\mathrm{C}_{10} \mathrm{H}_{16} \mathrm{O}$ & 152 & 3.14 & 0.25 & - & - \\
\hline 60 & 1253 & Carvone & $\mathrm{C}_{10} \mathrm{H}_{14} \mathrm{O}$ & 150 & 3.30 & 0.26 & 0.69 & 0.14 \\
\hline 61 & 1294 & (E)-Anethole & $\mathrm{C}_{10} \mathrm{H}_{12} \mathrm{O}$ & 148 & - & - & 7.50 & 1.53 \\
\hline 62 & 1304 & 1H-Indole & $\mathrm{C}_{8} \mathrm{H}_{7} \mathrm{~N}$ & 117 & 7.74 & 0.62 & - & - \\
\hline 63 & 1323 & 2-Methoxy-4-vinylphenol & $\mathrm{C}_{9} \mathrm{H}_{10} \mathrm{O}_{2}$ & 150 & 20.06 & 1.60 & - & - \\
\hline 64 & 1357 & a-Cubebene & $\mathrm{C}_{15} \mathrm{H}_{24}$ & 204 & - & - & 1.69 & 0.34 \\
\hline 65 & 1366 & Eugenol & $\mathrm{C}_{10} \mathrm{H}_{12} \mathrm{O}_{2}$ & 164 & 3.30 & 0.26 & - & - \\
\hline 66 & 1385 & a-Copaene & $\mathrm{C}_{15} \mathrm{H}_{24}$ & 204 & 30.60 & 2.44 & 10.52 & 2.15 \\
\hline 67 & 1392 & Modhephene & $\mathrm{C}_{15} \mathrm{H}_{24}$ & 204 & 7.34 & 0.59 & 1.32 & 0.27 \\
\hline 68 & 1409 & (Z)-Jasmone & $\mathrm{C}_{11} \mathrm{H}_{16} \mathrm{O}$ & 164 & 7.41 & 0.59 & 0.99 & 0.20 \\
\hline 69 & 1433 & $\beta$-Caryophyllene & $\mathrm{C}_{15} \mathrm{H}_{24}$ & 204 & 97.04 & 7.74 & 33.20 & 6.79 \\
\hline 70 & 1441 & B-Copaene & $\mathrm{C}_{15} \mathrm{H}_{24}$ & 204 & 7.40 & 0.59 & 1.29 & 0.26 \\
\hline 71 & 1455 & Coumarin & $\mathrm{C}_{9} \mathrm{H}_{6} \mathrm{O}_{2}$ & 146 & 26.88 & 2.14 & 15.63 & 3.20 \\
\hline 72 & 1458 & Neryl acetone & $\mathrm{C}_{13} \mathrm{H}_{22} \mathrm{O}$ & 194 & 4.79 & 0.38 & - & - \\
\hline 73 & 1461 & (E)- $\beta$-Famesene & $\mathrm{C}_{15} \mathrm{H}_{24}$ & 204 & 15.85 & 1.26 & 4.74 & 0.97 \\
\hline 74 & 1467 & a-Humulene & $\mathrm{C}_{15} \mathrm{H}_{24}$ & 204 & 16.31 & 1.30 & 3.58 & 0.73 \\
\hline 75 & 1488 & ४-Muurolene & $\mathrm{C}_{15} \mathrm{H}_{24}$ & 204 & 47.18 & 3.76 & 18.72 & 3.83 \\
\hline 76 & 1494 & Germacrene D & $\mathrm{C}_{15} \mathrm{H}_{24}$ & 204 & 26.66 & 2.13 & 2.46 & 0.50 \\
\hline 77 & 1500 & B-Selinene & $\mathrm{C}_{15} \mathrm{H}_{24}$ & 204 & 52.23 & 4.16 & 48.90 & 10.01 \\
\hline 78 & 1514 & 8-Cadinene & $\mathrm{C}_{15} \mathrm{H}_{24}$ & 204 & 14.71 & 1.17 & 5.61 & 1.15 \\
\hline 79 & 1518 & S-Cadinene & $\mathrm{C}_{15} \mathrm{H}_{24}$ & 204 & 30.77 & 2.45 & 9.30 & 1.90 \\
\hline 80 & 1537 & (Z)-Nerolidol & $\mathrm{C}_{15} \mathrm{H}_{26}$ & 222 & 20.80 & 1.66 & - & - \\
\hline 81 & 1553 & Caryophyllene oxide & $\mathrm{C}_{15} \mathrm{H}_{24}$ & 220 & 234.16 & 18.67 & 195.44 & 39.99 \\
\hline \multicolumn{5}{|c|}{ Total } & $1,254.00$ & 100 & 488.74 & 100 \\
\hline
\end{tabular}

${ }^{11} \mathrm{RI}$, retention index.

${ }^{2} \mathrm{MF}$, molecular formula.

${ }^{3} \mathrm{MW}$, molecular weight

4)I.S, internal standard. 
Table 3. Relative contents of the functional groups in the volatile compound detected in Artemisia annua L. by different extraction method

\begin{tabular}{|c|c|c|c|c|c|c|}
\hline \multirow{2}{*}{ Functional groups } & \multicolumn{3}{|c|}{ SDE } & \multicolumn{3}{|c|}{ SPME } \\
\hline & No. & Area\% & $\mathrm{mg} / \mathrm{kg}$ & No. & Area $\%$ & $\mathrm{mg} / \mathrm{kg}$ \\
\hline Alcohol & 27 & 26.81 & 336.18 & 7 & 8.23 & 40.23 \\
\hline Aldehyde & 13 & 2.06 & 25.89 & 5 & 1.16 & 6.14 \\
\hline Ester & 3 & 2.09 & 26.22 & 1 & 0.15 & 0.74 \\
\hline Hydrocarbon & 22 & 31.12 & 390.30 & 18 & 30.76 & 150.34 \\
\hline Ketone & 12 & 18.62 & 233.51 & 7 & 19.61 & 95.85 \\
\hline Oxide & 1 & 18.67 & 234.16 & 1 & 39.99 & 195.44 \\
\hline N-containing compound & 1 & 0.62 & 7.74 & - & - & \\
\hline Total & 79 & 100.00 & $1,254.00$ & 39 & 100 & 488.74 \\
\hline
\end{tabular}

$488.74 \mathrm{mg} / \mathrm{kg}$ 이 확인되었다. 개똥쑥에서 분석된 휘발성 향 기성분 중 oxide류 1종이 확인되었는데, $39.99 \%$ 로 가장 높 은 peak area를 나타내었다. 다음으로 hydrocarbon류가 18종 으로 $30.76 \%$ 를 차지하였으며, ketone류 7종(19.61\%), alcohol류 7종(8.23\%), aldehyde류 5종(1.16\%) 및 ester류 1종 $(0.15 \%)$ 순으로 확인되었다(Table 3$)$.

가장 높은 함량으로 동정된 휘발성 향기성분은 caryophyllene oxide로 함량은 $195.44 \mathrm{mg} / \mathrm{kg}$ 이었으며, camphor(68.93 $\mathrm{mg} / \mathrm{kg}), \quad \beta$-selinene $(48.90 \mathrm{mg} / \mathrm{kg}), \quad \beta$-caryophyllene $(33.20$ $\mathrm{mg} / \mathrm{kg}$ ), $\gamma$-muurolene $(18.72 \mathrm{mg} / \mathrm{kg})$, coumarin $(15.63 \mathrm{mg} / \mathrm{kg})$, 1,8-cineole(14.20 mg/kg), a-copaene $(10.52 \mathrm{mg} / \mathrm{kg}$ ) 및 $\delta$ -cadinene $(9.30 \mathrm{mg} / \mathrm{kg})$ 등 순으로 나타났다. Camphor의 상 대적 농도는 $14.10 \%$ 로 $\mathrm{SDE}$ 법에 의해 확인된 peak area 보다 더 높게 동정되었다. B-Selinene은 herbal한 특성으로 셀러 리와 천궁에서 동정된 바(28) 있으며, $\mathrm{SPME}$ 법에서 $10.01 \%$ 로 SDE법 보다 높게 확인되었다. $\gamma$-Muurolene은 oily 및 herbal한 특성을 가진 sesquiterpene으로 인진쑥 정유 성분으 로 동정된 바 있으며, coumarin은 국내에서 자생하고 있는 쑥속 식물의 정유성 분으로 비접촉성 증기상태에서도 높은 항균효과가 있다고 보고되었다(29). (E)-Pinocarveol, pinocarvone 및 borneol은 대둔산 더위지기의 주요 성분으 로 동정되었는데(26), 본 연구에서 각각의 함량은 7.53 $\mathrm{mg} / \mathrm{kg}, 7.27 \mathrm{mg} / \mathrm{kg}$ 및 $6.46 \mathrm{mg} / \mathrm{kg}$ 으로 동정되어 개똥쑥의 주요 휘발성 향기성분으로 확인되었다. Cadinene은 green, sweet 및 herbal한 특징으로 $a, \beta, \gamma, \delta$ 형태의 4 가지 이성체가 존재하고 있으며, 개똥쑥에서는 y 및 $\delta$ 형태가 각각 $1.15 \%$ 및 $1.90 \%$ 로 확인되었다. Copaene은 woody 및 earthy한 특징 을 가지며, 개똥쑥에서 a-copaene 및 $\beta$-copaene이 동정되었 으며, 상대적 농도는 $2.15 \%$ 및 $0.26 \%$ 로 $\mathrm{SPME}$ 법이 $\mathrm{SDE}$ 법 보다 낮게 확인되었다.

\section{추출방법에 따른 개똥쑥의 휘발성 향기성분 비교}

$\mathrm{SDE}$ 및 $\mathrm{SPME}$ 법에 따라 추출하여 확인된 개똥쑥의 휘발 성 향기성분은 $\mathrm{SDE}$ 법에서 79 종으로 함량은 $1,254.00 \mathrm{mg} / \mathrm{kg}$
이었으며, $\mathrm{SPME}$ 법에서는 39종으로 함량은 $488.74 \mathrm{mg} / \mathrm{kg}$ 으로 동정된 화합물의 구성과 함량에 대한 유의적인 차이가 있었다. 동정된 개똥쑥의 휘발성 주요 향기성분은 terpenoid 류로 추출 방법에 따른 peak area는 $\mathrm{SDE}$ 법에서 $91.09 \%$, SPME법에서 $95.50 \%$ 로 나타났다(Table 4). SDE법으로 추 출한 후 동정된 terpenoid류 중 oxygenated monoterpene류가 $39.63 \%(497.01 \mathrm{mg} / \mathrm{kg})$ 가장 높게 확인되었으며, 다음으로 sesquiterpene hydrocarbon류 $27.60 \%(346.08 \mathrm{mg} / \mathrm{kg})$, oxygenated sesquiterpene류 $20.33 \%(254.96 \mathrm{mg} / \mathrm{kg})$, monoterpene hydrocarbon류 $3.53 \%(44.23 \mathrm{mg} / \mathrm{kg})$ 순으로 확인되었다 (Table 4). SPME법에서는 oxygenated sesquiterpene류가 $39.99 \%(195.44 \mathrm{mg} / \mathrm{kg})$ 로 가장 높게 동정되었으며, 다음으 로 sesquiterpene hydrocarbonn 류가 $28.91 \%(141.32 \mathrm{mg} / \mathrm{kg})$, oxygented monotepene류가 $24.75 \%(120.96 \mathrm{mg} / \mathrm{kg})$ 및 monterpene hydrocarbon류가 $1.85 \%(9.02 \mathrm{mg} / \mathrm{kg})$ 순으로 나 타났다(Table 4). 개똥쑥의 주요 휘발성 향기성분인 terpenoid 류는 Mevalonic acid(MVA) pathway 및 Methylerythritol phosphate(MEP) pathway의 생합성 경로를 통해서 생성되 어진다(30).

$\mathrm{SDE}$ 및 $\mathrm{SPME}$ 법에서 공통으로 동정된 주요 휘발성 향기 성분은 caryophyllene oxide, $\beta$-caryophyllene, camphor, $\beta$ -selinene, $\gamma$-muurolene, 1,8-cineol, (E)-pinocarveol, pinocarvone, borneol, coumarin, a-copaene, $\beta$-copaene, germacrene $\mathrm{D}, \gamma$ -cadinene, $\delta$-cadinene, (E)- $\beta$-farnesene, a-humulene, (Z)jasmone 및 modhephene 등이 확인되었다.

Germacrene은 다양한 식물에 함유되어 있는 휘발성 화합 물로 $\mathrm{A}, \mathrm{B}, \mathrm{C}, \mathrm{D}, \mathrm{E}$ 형 5 개의 이성체가 있으며, 열수 추출과정 중에 생성되는 성분으로 보고된 바 있는데(31), 본 연구에서 는 SDE 및 SPME법 모두에서 germacrene $\mathrm{D}$ 가 확인되었다. Germacrene $\mathrm{D}$ 의 휘발성 향기성분 상대적 농도는 $\mathrm{SDE}$ 법에 서 $2.13 \%, \mathrm{SPME}$ 법에서는 $0.5 \%$ 로 동정되어 연속수증기증 류추출방법에서 좀 더 많은 함량을 확인할 수 있었다.

$\mathrm{SDE}$ 법에서만 동정된 휘발성 향기성분은 sabinene, $\beta$ -pinene, a-terpinene, $\gamma$-terpinene, yomogi alcohol, myrtenol, 
Table 4. Relative contents of the functional groups in the terpene compound detected in Artemisia annua L. by different extraction method

\begin{tabular}{ccccccc}
\hline \multirow{2}{*}{ Functional groups } & \multicolumn{3}{c}{ SDE } & \multicolumn{3}{c}{ SPME } \\
\cline { 2 - 7 } & No. & $\mathrm{mg} / \mathrm{kg}$ & Area\% & No. & $\mathrm{mg} / \mathrm{kg}$ & Area\% \\
\hline monoterpene hydrocarbons & 11 & 44.23 & 3.53 & 6 & 9.02 & 1.85 \\
oxygenated monoterpene & 23 & 497.01 & 39.63 & 13 & 120.96 & 24.75 \\
sesquiterpene hydrocarbons & 11 & 346.08 & 27.60 & 12 & 141.32 & 28.91 \\
oxygenated sesquiterpene & 2 & 254.96 & 20.33 & 1 & 195.44 & 39.99 \\
\hline Total & 47 & $1,142.28$ & 91.09 & 32 & 466.74 & 95.50 \\
\hline
\end{tabular}

(A)
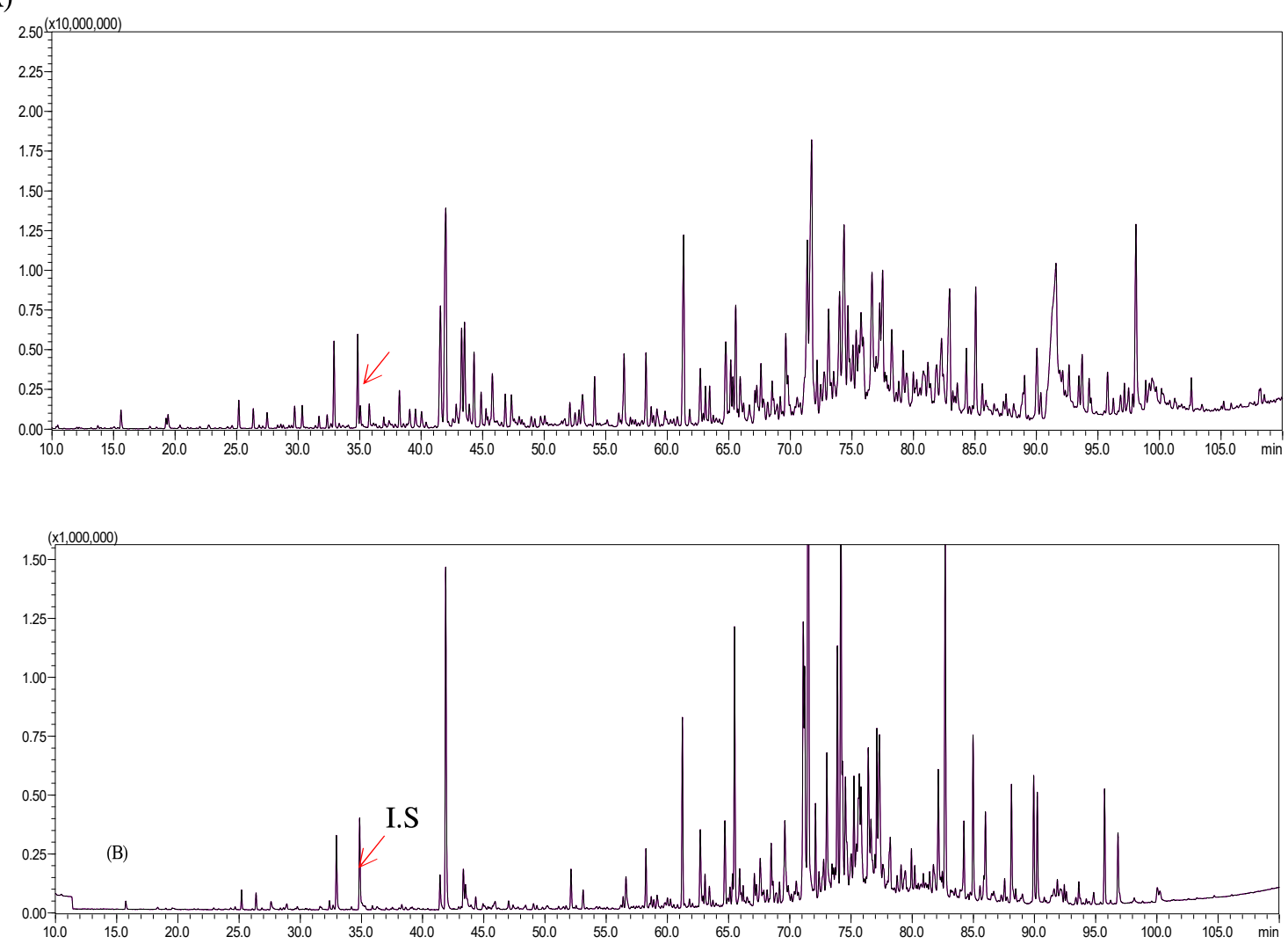

Fig 1. GC-MS chromatograms of the volatile flavor compounds in Artemisia annua L. by (A) simultaneous steam distillation extraction (SDE) \& (B) solid phase microextraction (SPME).

I.S, internal standard.

(Z)-nerolidol, 2-methoxy-4-vinylphenol, p-cymen-8-ol, eugenol, a-terpinolene, a-campholenal, camphene hydrate, (Z)-chrysanthenol, phenethyl alcohol, (Z)-sabinene hydrate 등이 확인되었다. Eugenol은 정향(clove)의 주요 휘발성 향 기성분으로 알려져 있는데, cycloxygenase 및 lipoxygenase 의 작용을 방해하여 신경전달물질 방출을 억제하여 통증 작용을 나타내는 감각 수용체의 반응 억제로 진통 효과를 나타내는 성분으로 사자발쑥 및 황해쑥에서 동정된 바 있다 $(29,32)$. 본 실험에서는 $\mathrm{SDE}$ 법에서만 $0.26 \%$ 로 함량은 3.30 $\mathrm{mg} / \mathrm{kg}$ 으로 확인되었다. SPME법에서만 동정된 성분은 (E)-anethole 및 a-cubebene이었으며, 함량은 $7.50 \mathrm{mg} / \mathrm{kg}$ 및 $1.69 \mathrm{mg} / \mathrm{kg}$ 으로 확인되었다. Anethole은 sweet, herbal 및 anise한 특성을 가지며, 캐모마일차의 향기성분으로 소량 동정된 바 있으며 비누, 술 및 구강제 등의 향료로 사용되고 있다(33). Mild waxy 및 woody한 특성의 a-cubebene은 향모 정유 및 황칠나무 정유에 가장 많이 함유되어 있다고 보고 된 바(34) 있는데, 개똥쑥에서 $1.69 \mathrm{mg} / \mathrm{kg}$ 로 확인되었다. 결론적으로 추출방법에 따라 향기성분의 차이를 확인할 
수 있었으며, $\mathrm{SDE}$ 법과 $\mathrm{SPME}$ 법의 장점을 최대화하여 다각 적인 분석방법 적용이 필요하다고 판단된다.

\section{요 약}

본 연구는 개똥쑥을 이용하여 기능성 식품 개발을 위한 기초자료를 제공하고자 추출방법에 따른 휘발성 향기성분 을 비교하였다. 개똥쑥의 휘발성 향기성분은 SDE 및 SPME 법을 이용하여 추출한 후 GC-MS로 분석하였다. 개똥쑥의 휘발성 향기성분은 $\mathrm{SDE}$ 법과 $\mathrm{SPME}$ 법에서 각각 79종 및 39종의 화합물로 분리 동정되었다. 총 함량은 각각 $1,254.00$ $\mathrm{mg} / \mathrm{kg}$ 및 $488.74 \mathrm{mg} / \mathrm{kg}$ 으로 확인되었다. SDE법에서 분리 동정된 성분들은 alcohol류 27종으로 다수를 차지하였고, aldehyde류 13종, ester류 3종, hydrocarbon 22종, ketone류 12종, oxide류 1종 및 n-containing compound류 1종 동정되 었다. SPME법에서는 alcohol류 7종, aldehyde류 5종, ester류 1종, hydrocarbon류 18종, ketone류 7종 및 oxide류 1종이 확인되었다. 개똥쑥의 주요 휘발성 향기성분은 caryophyllene oxide, $\beta$-caryophyllene, camphor, $\beta$-selinene, $\gamma$-muurolene, 1,8-cineol, (E)-pinocarveol 및 pinocarvone 등이 확인되었으 며, 가장 많은 함량으로 동정된 성분은 caryophyllene oxide 로 $\mathrm{SDE}$ 법은 $234.16 \mathrm{mg} / \mathrm{kg}$, SPME법에서 $195.44 \mathrm{mg} / \mathrm{kg}$ 으로 확인되었다. Sabinene, $\beta$-pinene, a-terpinene, $\gamma$-terpinene, yomogi alcohol, myrtenol, (Z)-nerolidol, p-cymen-8-ol 및 eugenol은 $\mathrm{SDE}$ 법에서 동정된 성분이며, (E)-anethole 및 a -cubebene은 SPME법에서만 확인되었다. 결과적으로 추출 방법에 따른 휘발성 향기성분의 조성과 함량의 차이를 확인 할 수 있었으며, SDE법에 의한 경우가 SPME법보다 더 많은 향기성분이 동정되었다.

\section{References}

1. Hwang YM, Kim MK, Jung MS (2006) Analysis of volatile flavor compounds from Artemisia capillaris using SPME. Ann Plant Resour Res, 5, 17-31

2. Yook CS (1997) Medicinal botany. Jinmyung pub, Seoul, Korea, p 293

3. Song HN (2013) Quality properties of fermented mugworts and the rapid pattern analysis of their volatile flavor components via surface acoustic wave (SAW) based electronic nose sensor in the GC system. Korean J Food Preserv, 20, 554-563

4. Kim OC, JEAN HJ (1994) Volatile components Artemisia apiaceae Herba. Agric Chem Biotech, 37, 37-42

5. Ryu JH, Kim RJ, Lee SJ, Kim IS, Lee HJ, Sung NJ
(2011) Nutritional properties and biological activities of Artemisia annua L.. J Korean Soc Food Sci Nutr, 40, 163-170

6. Kim HC, Kil BS, Lee YH (2001) The antifungal activity of chemical substances from Artemisia annua. Korean J Ecol, 24, 137-140

7. Klayman DL (1985) Qinghaosu (artemisinin): an antimalarial drug from China. Science, 228, 1049-1055

8. Ryu JH, Lee SJ, Kim MJ, Shin JH, Kang SK, Cho KM, Sung NJ (2011) Antioxidant and anticancer activities of Artemisia annua L. and determination of functional compounds. J Korean Soc Food Sci Nutr, 40, 509-516

9. Choi KS, Choi BY, Park HK, Kim JH, Park JS, Yoon CN (1998) Flavor components of Artemisia Lavandulaefolia DC. Korean J Food Sci Technol, 20, 774-779

10. Jang SB, Chang SY, Inn MK, Jeong JH, Yook CS (2000) Studies on the components of essential oils in the aerial part of Artemisia mongolica. Bull K H Pharm Sci, 28, 45-49

11. Lee JM, Lee JM, Lee GC, Bock JY, Hwang KJ (2010) Comparison of the volatile flavor compounds in different tobacco types by different extraction methods. J Korean Soc Tob Sci, 32, 77-87

12. Song YE, Ku CS, Mun SP, Ryu JS, Kim DH, Choi JS, Choi YG (2002) Volatile aroma compounds and their characteristics of Labiatae by solid-phase microextraction (SPME). Korean J Med Crop Sci, 10, 120-125

13. Chung MS, Lee MS (2003) Analysis of volatile compounds in Elsholtzia splendens by solid phase microextraction. Korean J Soc Food Cookery Sci, 19, 79-82

14. Schultz TH, Flath RA, Mon TR, Eggling SB, Teranishi R (1977) Isolation of volatile components from a model system. J Agric Food Chem, 25, 446-449

15. Hong YS, Lee YS, Kim KS (2017) Comparison of volatile flavor compounds of yuzu, kumquat, lemon and lime. Korean J Food Preserv, 24, 394-405

16. Yang D, Michel L, Chaumont JP, Millet-Clerc J (1999) Use of caryophyllene oxide as an antifungal agent in an in vitro experimental model of onychomycosis. Mycopathologia, 148, 79-82

17. Sensch O, Vierling W, Brandt W, Reiter M (2000) Effects of inhibition of calcium and potassium currents in guinea-pig cardiac contraction: comparison of $\beta$ -caryophyllene oxide, eugenol, and nifedipine. $\mathrm{Br} \mathrm{J}$ Pharmacol, 131, 1089-1096 
18. Akahoshi, G (1983) Koryonogakkak. Taenippondosyo Tokyo, Japan, p 299-315

19. Boland DJ, Brophy JJ, House APN (1991) Eucalyptus leaf oils: use, chemistry, distillation and marketing. Inkata Press, Melbourne and Sidney, Australia, p 252

20. Chung MS, Lee MS (1998) Analysis of volatile flavor components of Plearospermun kamtschaticum. Korean J Soc Food Sci, 14, 541-546

21. Arctander S (1969) Perfume and flavor chemicals. Montclair, NJ, USA, p 150

22. Juteau F, Masotti V, Bessiere JM, Dherbomez M, Viano J (2002) Antibacterial and antioxidant activities of Artemisia annua essential oil. Fitoterapia, 73, 532-535

23. Woerdenbag HJ, Bos R, Salomons MC, Hendriks H, Pras N, Malingre, TM (1993) Volatile constituents of Artemisia annua L. (Asteraceae). Flavour and Fragrance $\mathrm{J}, 8,131-137$

24. Khangholil S, Rezaeinodehi A (2008) Effect of drying temperature on essential oil content and composition of sweet wormwood (Artemisia annua) growing wild in Iran. Pak J Biol Sci, 11, 934-937

25. Choi EC (2017) A study on skin physiological activities and clinical effects of Artemisia annua L. essential oil. $\mathrm{Ph}$ D Thesis, Hoseo University, Korea, p 18-19

26. Hong CU (2004) Comparison of essential oil composition of Artemisia iwayomogi and Artemisia capillaris. J Korean Soc Appl Biol Chem, 47, 124-129

27. Hwang DI, Won KJ, Kim DY, Yoon SW, Park JH, Kim BK, Lee HM (2016) Anti-adipocyte differentiation activity and chemical composition of essential oil from Artemisia annua. Nat Prod Commun, 11, 539-542

28. Choi SH, Kim HJ (2000) The flavor components of Korean Cnidium officinale Makino. J Korean Soc Food Sci Nutr, 29, 582-585

29. Cho YH, Chiang MH (2001) Essential oil composition and antibacterial activity of Artemisia capillaris, Artemisia argyi, and Artemisia princeps. J Korean Int Agric, 13, 313-320

30. Bowles EJ (2003) The chemistry of aromatherapeutic oils. Allen \& Unwin, Sydeney, Australia, p 27-39

31. Lee YS (2005) Development of aroma oils from Gangwon native plants. MS Thesis, Kangwon National University, Korea, p 54

32. Battaglia S (2004) The complete guide to aromatherapy. Perfect Potion, Canberra, Australia, p 5-10

33. Choi SH (2008) Volatile aroma components of hibiscus herb tea. J Korea Tea, 14, 195-204

34. Yeon BR, Jeong MS, Noh HS, Kim SM (2011) Volatile fragrant chemicals in Hyangmo (Hierochloe odorata (L). P. Beauv.). J Agric Life Environ Sci, 23, 30-34 